\title{
Cálculo da Segunda Hiperpolarizabilidade da L-arginina Fosfatada Monohidratada Considerando o Efeito do Ambiente Cristalino
}

\author{
Rosemberg Fortes Nunes Rodrigues, Clodoaldo Valverde, Basílio Baseia \& \\ Heibbe C. B. de Oliveira
}

\section{Introdução}

Para uma classe de matérias com alta aplicabilidade em óptica moderna estão os materiais com características Óptica não Linear (ONL), no qual os cristais híbridos orgânicos-inorgânicos se mostraram excelentes candidatos para a sua confecção [1]. Estes sistemas podem exibir elevado comportamento óptico não linear na região do visível e boa estabilidade térmica e mecânica, quando comparados com os cristais orgânicos. Um típico cristal orgânico-inorgânico ONL é a L-arginina fosfatada monohidratada $\left(\mathrm{C}_{6} \mathrm{H}_{14} \mathrm{~N}_{4} \mathrm{O}_{2} \mathrm{H}_{3} \mathrm{PO}_{4} \mathrm{H}_{2} \mathrm{O}\right)$, mais conhecida pela sigla (LAP) [2,3]. A sua unidade cristalográfica assimétrica é composta por uma molécula orgânica quiral [L-arginina]+, uma molécula inorgânica $\left[\mathrm{H}_{2} \mathrm{PO}_{4}\right]-$, e uma molécula de água.[3].

A estrutura cristalina da LAP pode ser descrita como camadas alternadas de íons de fosfato, íons L-arginina e moléculas de água presas por ligações intermoleculares de hidrogênio de uma forma rígida não-centrossimétrica [2]. A estrutura molecular da LAP é mostrada na figura 13.

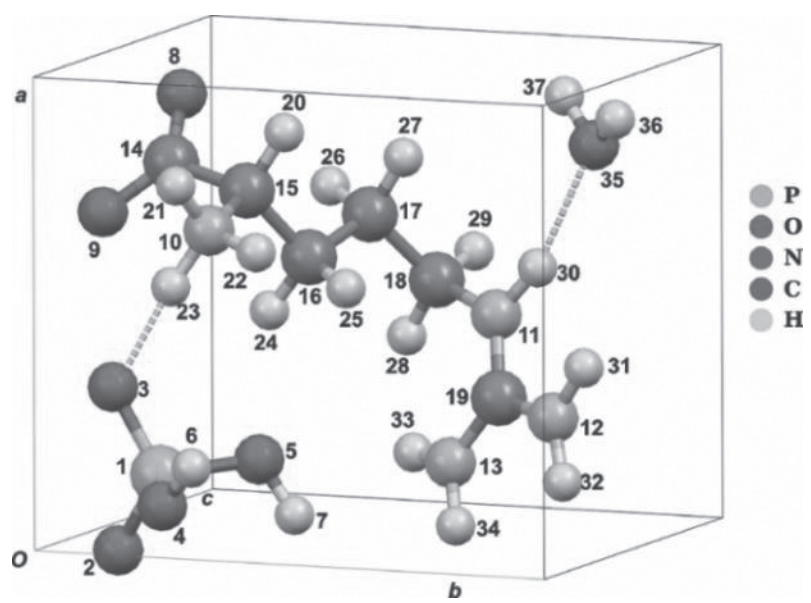

Figura 1. Estrutura molecular da unidade assimétrica da LAP4)

No estado sólido, a LAP se cristaliza no sistema monoclínico, que pertence ao grupo de simetria espacial $\mathrm{P} 2{ }_{1}$, e os parâmetros da cela unitária são $\mathrm{a}=7,319 \AA$, $\mathrm{b}=$ $7,912 \AA, c=10,779 \AA, \beta=98,05^{\circ}$ (ângulo cristalográfico entre os eixos a e c) [3]. Observa-se ainda a existência de duas unidades assimétricas por cela unitária. No presente 
trabalho empregamos um modelo teórico proposto por Fonseca et al. [4] para explorar as propriedades cristalinas da L-arginina fosfatada monohidratada. O objetivo é obter a segunda hiperpolarizabilidade dessa molécula envolvida em um ambiente cristalino, para este fim, utilizou-se a teoria do funcional da densidade (DFTDensity Functional Theory).

\section{Métodos}

Nesse trabalho foi feita uma abordagem supramolecular através da qual é possível detectar os efeitos da polarização do meio sobre as propriedades elétricas da LAP. Nesse aspecto, as moléculas vizinhas são vistas como cargas pontuais. Esta aproximação se baseia no fato de que as interações entre moléculas são, predominantemente, de natureza eletrostática e leva em conta os efeitos eletrostáticos de longo alcance [5].

A figura 2 mostra uma molécula de LAP envolvida por outras iguais. Nesse trabalho foi considerado um número próximo de 249 unidades assimétricas como cargas pontuais, constituindo um conjunto de celas unitárias $5 \times 5 \times 5$, e cada cela unitária contendo duas unidades assimétricas.

Os cálculos foram feitos nos nível DFT usando o conjunto de funções base 6-311+G(d), para os seguintes funcionais de densidade: B3LYP, B2PLYP, CAM-B3LYP.

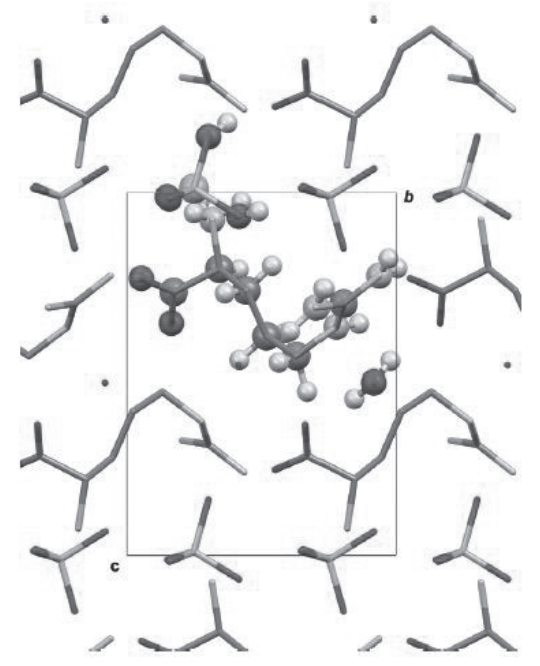

Figura 2. Projeção ao longo do eixo a do cristal mostrando a unidade assimétrica de LAP envolvida no campo de polarização das moléculas das unidades envolvidas tratadas como cargas pontuais4.

\section{Resultados e Discussão}

Inicialmente o cálculo das propriedades elétricas do cristal de LAP foi efetuado por Fonseca et al. [4], no nível MP2 utilizado o conjunto de funções base 6-311+G(d). Nesse trabalho as propriedades elétricas calculadas foram momento de dipolo, polarizabilidade linear e primeira hiperpolarizabilidade.

No presente trabalho, realizamos os cálculos das propriedades elétricas do cristal de LAP em nível DFT utilizando os funcionais, CAM-B3LYP, B3LYP e B2PLYP, e com o conjunto de funções base $6-311+G(d)$.

Ao comparar os valores do momento de dipolo elétricos da molécula envolvida no nível DFT, utilizando diferentes funcionais, com o valor invariável de 33,26 D (MP2), obtêm-se percentuais variados. Desse modo o CAM-B3LYP, nota-se uma diferença de $0,21 \%$. Assim, seguindo o mesmo raciocínio comparativo, verificam-se que, para B3LYP, tem-se $0,75 \%$ e por último, B2PLYP uma diferença de $0,45 \%$.

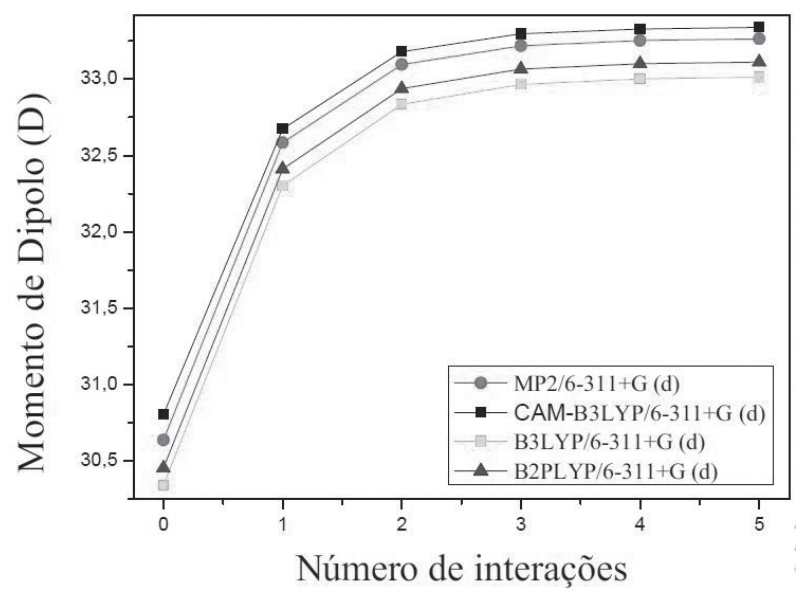

Figura 3. Evolução dos valores do momento de dipolo da LAP com os respectivos números de interação (conjunto cela unitária 5 X5X5)

Para o cálculo da segunda hiperpolarizabilidade utilizou-se o funcional CAM-B3LYP e B2PLYP, pois ambos representaram valores próximos ao MP2 no cálculo do momento de dipolo, com o objetivo de mostrar os valores de $(\gamma)$, notando uma rápida convergência assim como para o momento de dipolo. 
A tabela 1 mostram os resultados das componentes e resultante para segunda hiperpolarizabilidade $(\gamma)$ da LAP com os respectivos números de interação e uma visualização alternativa dessa convergência fica evidente através de gráficos da figura 3

Tabela 1. CAM-B3LYP/6-311+G(d) Resultados das componentes da segunda hiperpolarizabilidade e sua resultante (10-36esu)

\begin{tabular}{c|c|c|c|c|c|c}
\hline $\begin{array}{c}\text { Número de } \\
\text { Interações }\end{array}$ & 0 & 1 & 2 & 3 & 4 & 5 \\
\hline$\gamma_{x x x x x}$ & 25,97 & 18,65 & 18,13 & 18,65 & 18,68 & 11,36 \\
$\gamma_{y y y y}$ & 29,60 & 17,27 & 16,98 & 17,02 & 17,05 & 18,58 \\
$\gamma_{z z z z}$ & 20,70 & 8,93 & 7,92 & 7,72 & 7,70 & 12,06 \\
$\gamma_{x x y y}$ & 9,00 & 6,11 & 5,96 & 6,11 & 6,14 & 5,49 \\
$\gamma_{x x z z}$ & 6,63 & 4,25 & 4,12 & 4,14 & 4,15 & 4,08 \\
$\gamma_{y y z z}$ & $-4,44$ & $-0,66$ & $-0,59$ & $-0,47$ & $-0,48$ & 2,64 \\
$\gamma_{\text {total }}$ & 25,10 & 14,75 & 14,21 & 14,33 & 14,35 & 14,37 \\
\hline
\end{tabular}

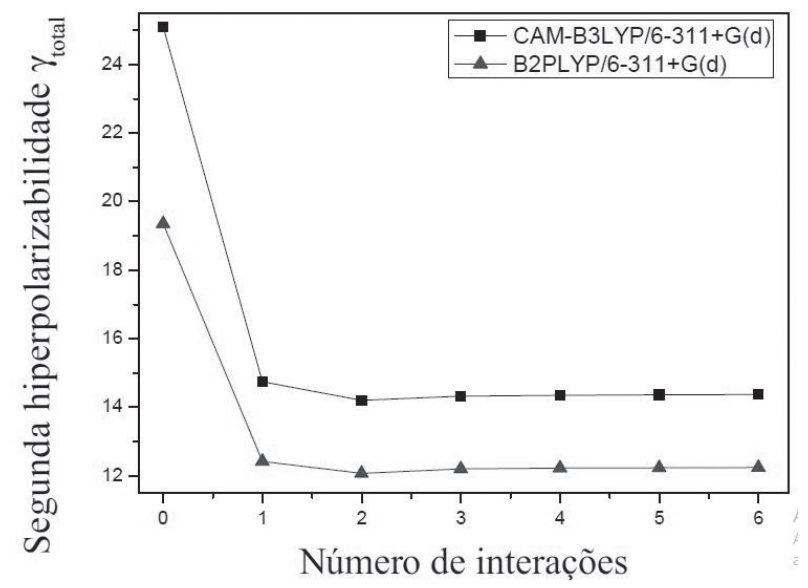

Figura 2. CAM-B3LYP e

\section{Conclusões}

Dessa forma os resultados apresentados para $(\gamma)$ serão de fundamental importância para o meio científico, pois o entendimento das propriedades ópticas dos materiais propicia a obtenção das maneiras de manipulação da luz. As investigações sobre essas propriedades e esse tipo material são vitais à aplicação da óptica moderna.

\section{Agradecimentos}

Os autores são gratos pelo apoio dado por FAPEG, CAPES e CNPQ.

\section{Referências}

1. S. Manivannan, S. Dhanuskodi, K. Kirschbaum; S. K. Tiwari, Cryst. Growth Des. 5, 1463, (2005).

2. K. Aoki, K. Nagano, Y. Litaka, Acta Crystallogr., Sect. B: Struct. Crystallogr. Cryst. Chem. 27, 11, (1971).

3. E. Espinosa, C. Lecomte, E. Molins, S. Veintemillas, A. Cousson, W. Paulus, Acta Crystallogr., Sect. B: Struct. Sci. 52, 519, (1996).

4. T. L. Fonseca, J.R. Sabino, M. A. Castro, H. C. Georg, J. Chem. Phys. 133, 144103, (2010).

5. M. B. Kanoun, E. Botek, B. Champagne, Chem. Phys. Lett. 487, 256, (2010).

\section{Rosemberg Fortes Nunes Rodrigues ${ }^{a, b}$, Clodoaldo Valverde ${ }^{a, b}$ *, Basílio Baseiac, Heibbe Cristhian Benedito de Oliveira $^{d}$}

${ }^{\text {a }}$ Campus Henrique Santillo, Universidade Estadual de Goiás, Rod. BR 153, km 98, 75.132-903 Anápolis, GO, Brasil.

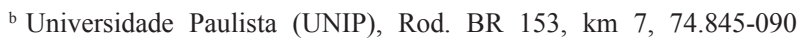
Goiânia, GO, Brasil

c Instituto de Física, Universidade Federal de Goiás, 74.001-970 Goiânia, GO, Brasil.

d Laboratório de Modelagem de Sistemas Complexos, Instituto de Química, Universidade de Brasília, 70904-970 Brasília, Brasil.

*E-mail: valverde@ueg.br 